\title{
COMPARACIÓN DE LA ESTRUCTURA Y DE LA FUNCIÓN DE LOS MIEMBROS ANTERIOR Y POSTERIOR DE Cuniculus taczanowskif Y Dinomys branickii
}

\section{COMPARISON OF THE FORE AND HINDLIMB STRUCTURE AND FUNCTION OF Cuniculus taczanowskii AND Dinomys branickii}

Karin Osbahr ${ }^{1}$

Pedro Acevedo ${ }^{2}$ Andrea Villamizar ${ }^{3}$ Daniela Espinosa ${ }^{3}$

\section{RESUMEN}

Las adaptaciones anatómicas que capacitan a los animales para desarrollarse de una manera específica en un ambiente determinado, se ven reflejadas en cambios morfológicos estructurales y fisiológicos. Cuniculus taczanowskii y Dinomys branickii son especies simpátricas, por lo cual,surgió la pregunta de si existen diferencias y similitudes en el esqueleto apendicular que expliquen sus hábitos locomotores, en el contexto de la ecología funcional. El análisis de las medidas óseas de los huesos largos que conforman el esqueleto apendicular muestra claras diferencias tanto inter como intraespecíficas, que permiten inferir características específicas de $D$. branickii y $C$. taczanowskii, durante la locomoción y, por lo tanto, durante los patrones de

${ }^{1}$ Líder Grupo de Investigación en Fauna Silvestre. Universidad de Ciencias Aplicadas y Ambientales U.D.C.A. Dirección para correspondencia: Calle 222 No. 55-37 Bogotá - D.C. kosbahr@udca.edu.co

2 Docente Facultad de Medicina Veterinaria - Área Anatomía. Universidad de Ciencias Aplicadas y Ambientales U.D.C.A. pacevedo@udca.edu.co

${ }^{3}$ Médico Veterinario. Universidad de Ciencias Aplicadas y Ambientales U.D.C.A. avg5000@hotmail.com, dan2_19@hotmail.com uso del hábitat. Los índices morfológicos corroboran las características anatómicas, que explica la capacidad para cavar propia de $C$. taczanowskii y el desplazamiento plantígrado durante la marcha lenta, característico de D. branickii.

Palabras clave: Tinajo de páramo, Guagua loba, esqueleto apendicular, índices morfológicos, morfometría ósea.

\section{SUMMARY}

The anatomical adaptations that allow animals to develop in a specific way in a certain environment reflect structural and physiological changes. Cuniculus taczanowskii and Dinomys branickii are simpatric species, so the question arose if possible differences and similarities in the appendicular skeleton explain their locomotive habits in the context of functional ecology. The analysis of the long bone measures of the appendicular skeleton show inter and intraspecific differences that allow to assume specific characteristics of $D$. branickii and C. taczanowskii during the locomotion and therefore during the patterns of habitat use. The morphologic indices corroborate the anatomical characteristics that explain the fossorial activities of $C$. taczanowskii and the slow walking cursorial adaptation of $D$. branickii.

Key words: Mountain paca, Pacarana, appendicular skeleton, morphological index, bone scaling. 


\section{INTRODUCCIÓN}

La locomoción representa un elemento clave para la supervivencia de los vertebrados terrestres. Asociada a la búsqueda de alimento, fuentes de agua y de refugio, a la evasión de depredadores y a la interacción con otros individuos, constituye un factor importante durante los patrones de actividad. La capacidad y la velocidad locomotoras reflejan la habilidad de moverse en un hábitat, afectando, directamente, la posibilidad de acceder parcial o totalmente a todos sus componentes y modificando, así, la forma eficaz de explotar un área (Irschick, 2002). El esqueleto apendicular de los mamíferos refleja sus hábitos ecológicos y locomotores. Los roedores presenta características generales similares a otros grupos, con algunas adaptaciones convergentes con las de otros mamíferos adaptados a diferentes tipos de desplazamiento. Concretamente, para los roedores histricognatos adaptados para la carrera, se observan similitudes morfológicas con representantes de los órdenes Artiodactila, Perisodactila y Carnivora, en los cuales, extremidades más largas, corresponden a metapodiales más largos, mientras que las falanges permanecen relativamente cortas (Ginsburg, 1961; Christiansen, 1999; Hildebrand E Goslow, 2001).

Los roedores del suborden Hystricognatha sobresalen por una gran diversidad anatómica y ecológica. Debido a que por anatomía no se encuentran altamente especializados, registran un repertorio amplio en los estilos de locomoción caracterizándose, incluso, en algunos casos, por presentar más de un hábito de locomoción (Elissamburu E Vizcaíno, 2004; Weisbecker E Schmid, 2007).

Durante los últimos años, se han realizado estudios enfocados hacia la estructura, la función y la cinemática de los miembros de los mamíferos relacionando, a su vez, el comportamiento general de una o más especies en particular (Endo et al. 2001; Bacigalupe et al. 2002; Fletcher, 2007; Taborelli et al. 2007, Santori et al. 2005, 2008). Kimura (2003) argumenta que la diferenciación funcional entre el miembro anterior y posterior constituye una característica única del comportamiento locomotor de un animal. Como complemento, Elissamburu E Vizcaíno (2004) concluyen que el estudio de los huesos largos en roedores caviomorfos es relevante para distinguir las características particulares de los diferentes tipos de locomoción. El desempeño de las especies relativamente especializadas para vivir en determinado hábitat normalmente es mayor en relación al que presentan en otro tipo de hábitat. Estas diferencias, se reflejan en los diversos taxones que componen un ensamblaje determinado, donde se han reportado correlaciones interespecíficas entre el hábitat, la morfología y la habilidad locomotora, con diferentes grados de especialización. Por lo tanto, el desempeño locomotor es un indicador potencialmente importante de la diferenciación ecológica entre especies (Irschick, 2002).

Aunque el tinajo de páramo y la guagua loba son representantes de dos familias de roedores con características morfológicas propias, se encuentran agrupados dentro del suborden Hystrocognatha, que incluye a los caviomorfos suramericanos. La gran diversidad anatómica y ecológica del grupo permite realizar estudios comparativos y explorar algunas de las características funcionales reflejadas en el esqueleto apendicular (Loguercio, 2005; Weisbecker E Schmid, 2007, Rocha-Barbosa et al. 1996a,b; 2002; 2005; 2007). La longitud de los huesos largos es un indicador relevante para distinguir los grupos de locomoción en los Caviomorfos (Elissamburu E Vizcaíno, 2004). Weisbecker E Schmid (2007) describen, respectivamente, a C. taczanowskii y $D$. branickii como animales terrestres y trepadores, pero esta condición únicamente ha sido documentada mediante el estudio general del esqueleto autopodial sin incluir otros elementos óseos de los miembros que, igualmente, influyen en la locomoción. Por lo tanto, el propósito del presente trabajo fue el de buscar diferencias y similitudes en el esqueleto apendicular de las dos especies, que expliquen sus hábitos locomotores y permitan establecer criterios en el contexto de la ecología funcional.

\section{MATERIALES Y MÉTODOS}

Para obtener las medidas morfométricas del esqueleto apendicular anterior (húmero, ulna, radio y metacarpo) y posterior (fémur, tibia y metatarso), se utilizó el material depositado en la colección biológica de la Universidad de Ciencias Aplicadas y Ambientales U.D.C.A (Tabla 1). Los huesos, se midieron empleando un calibrador de 0-150mm marca KEX, de acuerdo a la metodología sugerida por Pinto et al. (2002), (Tabla 2), siguiendo, a su vez, las medidas óseas propuestas por Vizcaíno \& Milne (2002) y por Elissamburu E Vizcaíno (2004) (Figura 1). 
Tabla 1. Medidas morfométricas de los ejemplares depositados en la Colección Biológica de la U.D.C.A.

Dinomys branickii

\begin{tabular}{|c|c|c|c|c|c|c|}
\hline No colección & Sexo & Peso gr & (LT) mm & Msu (mm) & Psu (mm) & $\operatorname{Lco}(\mathrm{mm})$ \\
\hline UIFS 135 & $M$ & 9250 & 600 & 77,7 & 110,7 & 200 \\
\hline UIFS 144 & $M$ & 9700 & 670 & 69,4 & 108,9 & 210 \\
\hline UIFS 116 & $M$ & 9400 & 570 & 72,2 & 104,7 & 215 \\
\hline UIFS 147 & $\mathrm{M}$ & 9300 & 710 & 66,0 & 120,0 & 200 \\
\hline UIFS 138 & $\mathrm{H}$ & 7550 & 520 & 68,6 & 93,4 & 190 \\
\hline UIFS 062 & $\mathrm{M}$ & 9700 & 640 & 72,0 & 119,0 & 180 \\
\hline UIFS 060 & $\mathrm{H}$ & 8750 & 530 & 65,5 & 109,5 & 200 \\
\hline UIFS 061 & $\mathrm{H}$ & 9650 & 610 & 65,0 & 102,0 & 155 \\
\hline UIFS 160 & $\mathrm{H}$ & 7300 & 530 & 62,0 & 98,6 & 180 \\
\hline UIFS 140 & $\mathrm{M}$ & 10000 & 790 & 60,0 & 120,0 & 160 \\
\hline UIFS 021 & $\mathrm{M}$ & 10900 & 650 & 69,0 & 127,7 & 210 \\
\hline UIFS 143 & $\mathrm{H}$ & 7400 & 530 & 68,8 & 103,8 & 195 \\
\hline \multicolumn{2}{|l|}{ Promedio } & 9075 & 613 & 68,02 & 109,9 & 191 \\
\hline \multicolumn{2}{|c|}{ Desviación } & 1123 & 84 & 4,48 & 10,1 & 19,2 \\
\hline
\end{tabular}

Cuniculus taczanowskii

\begin{tabular}{|c|c|c|c|c|c|c|}
\hline UIFS 102 & $\mathrm{H}$ & 5000 & 600 & 53,6 & 96,4 & 5,0 \\
\hline UIFS 017 & $?$ & 5250 & 630 & 56,0 & 93,0 & 5,0 \\
\hline UIFS 158 & $\mathrm{H}$ & 4500 & 590 & 52,6 & 90,0 & 11,1 \\
\hline UIFS 005 & $\mathrm{H}$ & 3200 & 620 & 48,8 & 70,0 & 6,0 \\
\hline \multicolumn{2}{c|}{ Promedio } & 4488 & 610 & 52,8 & 87,4 & 6,8 \\
\hline \multicolumn{2}{|c|}{ Desviación } & 913 & 18,3 & 3,0 & 11,9 & 2,9 \\
\hline
\end{tabular}

(UIFS) Unidad de Investigación en Fauna Silvestre, (LT) Largo total (incluye cabeza y cola), (Msu) Mano sin uña, (Psu) Pata sin uña, (Lco) Longitud de la cola.

Para el análisis, se tuvieron en cuenta los datos obtenidos para los huesos de los miembros izquierdo y derecho.

Los valores estimados del largo del miembro anterior de cada especie, se obtuvieron sumando las longitudes fisiológicas del húmero, el radio y el tercer metacarpo. Para el largo del miembro posterior de cada especie, se realizó la suma de las longitudes fisiológicas del fémur, de la tibia y del tercer metatarso (Quesada E Gloobe, 1988).

Con el fin de relacionar las medidas obtenidas para determinar conceptos locomotores en estas dos especies, se emplearon los índices sugeridos por Vizcaíno et al. (1999), Vizcaíno E Milne (2002) y Elissamburu E Vizcaíno (2004):
- Índice braquial (IB): LFR/LFH x 100. Es un indicador de la extensión, donde el miembro anterior se adapta para tener movimientos más rápidos.

- Índice del movimiento del hombro (IMH): LTDH/LFH x 100. Registra la ventaja mecánica de los músculos deltoides y pectoral mayor, actuando a través de la articulación del hombro.

- Índice de la habilidad fosorial (IHF): LOU/(LMU-LOU) x 100. Define la ventaja mecánica de los músculos dorsoepitrocleares y tríceps en la extensión del codo; es considerado un buen indicador de la habilidad para cavar (Hildebrand et al. 1985; Vizcaíno et al. 1999; Fernández et al. 2000; Vizcaíno \& Milne, 2002).

- Índice epicondilar (IE): HADM/HLF x 100. Relaciona el ancho disponible para el origen de los músculos flexor, 
Tabla 2. Descripción de las medidas óseas utilizadas.

\begin{tabular}{|c|c|c|c|}
\hline HUESO & MEDIDA & SIGLA & DESCRIPCIÓN \\
\hline \multirow{4}{*}{ Húmero } & Anchura distal máxima & $\mathrm{ADMH}$ & $\begin{array}{l}\text { Largo que existe tomado del epicóndilo lateral al epicóndilo } \\
\text { medial. }\end{array}$ \\
\hline & Longitud fisiológica & LFH & $\begin{array}{l}\text { Sesde el extremo proximal de la cabeza del húmero hasta el } \\
\text { extremo distal de la tróclea. }\end{array}$ \\
\hline & Anchura mínima lateral de la diáfisis & AMLDH & $\begin{array}{l}\text { Ancho tomado desde la parte más angosta del cuerpo visto } \\
\text { frontalmente. }\end{array}$ \\
\hline & Largo tuberosidad deltoidea & LTDH & $\begin{array}{l}\text { Distancia desde el extremo proximal de la cabeza del humero } \\
\text { hasta el extremo distal de la tuberosidad deltoidea. }\end{array}$ \\
\hline \multirow{3}{*}{ Ulna } & Longitud máxima & LMU & $\begin{array}{l}\text { Desde el extremo proximal de la tuberosidad del olecranon } \\
\text { hasta el extremo distal de la apófisis estiloides. }\end{array}$ \\
\hline & Largo del olecranon & LOU & $\begin{array}{l}\text { Distancia que hay desde la superficie articular hasta el } \\
\text { extremo proximal de la ulna. }\end{array}$ \\
\hline & Anchura mínima lateral de la diáfisis & AMDU & $\begin{array}{l}\text { Ancho tomado desde la parte más angosta del cuerpo visto } \\
\text { frontalmente. }\end{array}$ \\
\hline Radio & Longitud fisiológica & LFR & $\begin{array}{l}\text { Desde la superficie articular proximal hasta la superficie } \\
\text { articular distal del radio. }\end{array}$ \\
\hline Metacarpos & Longitud fisiológica & LFMc & $\begin{array}{l}\text { Desde la superficie articular proximal hasta la superficie } \\
\text { articular distal del tercer metacarpo. }\end{array}$ \\
\hline \multirow{3}{*}{ Fémur } & Anchura mínima de la diáfisis & FAMD & $\begin{array}{l}\text { Ancho tomado desde la parte más angosta del cuerpo, visto } \\
\text { frontalmente. }\end{array}$ \\
\hline & Longitud fisiológica & LFF & $\begin{array}{l}\text { Desde el extremo proximal de la cabeza del fémur hasta el } \\
\text { extremo distal de la tróclea. }\end{array}$ \\
\hline & $\begin{array}{l}\text { Distancia de la cabeza del fémur hasta el } \\
\text { trocánter mayor }\end{array}$ & DLCTMF & $\begin{array}{l}\text { Desde el extremo proximal de la cabeza, hasta el borde distal } \\
\text { del trocánter mayor. }\end{array}$ \\
\hline \multirow{2}{*}{ Tibia } & Longitud fisiológica & LFT & $\begin{array}{l}\text { Desde el cóndilo lateral hasta la cavidad articular distal de la } \\
\text { tibia. }\end{array}$ \\
\hline & Anchura mínima de la diáfisis & AMDT & $\begin{array}{l}\text { El ancho tomado desde la parte más angosta del cuerpo, } \\
\text { visto frontalmente. }\end{array}$ \\
\hline Metatarsos & Longitud fisiológica & LFMt & $\begin{array}{l}\text { Desde la superficie articular proximal hasta la superficie } \\
\text { articular distal del tercer metatarso. }\end{array}$ \\
\hline
\end{tabular}



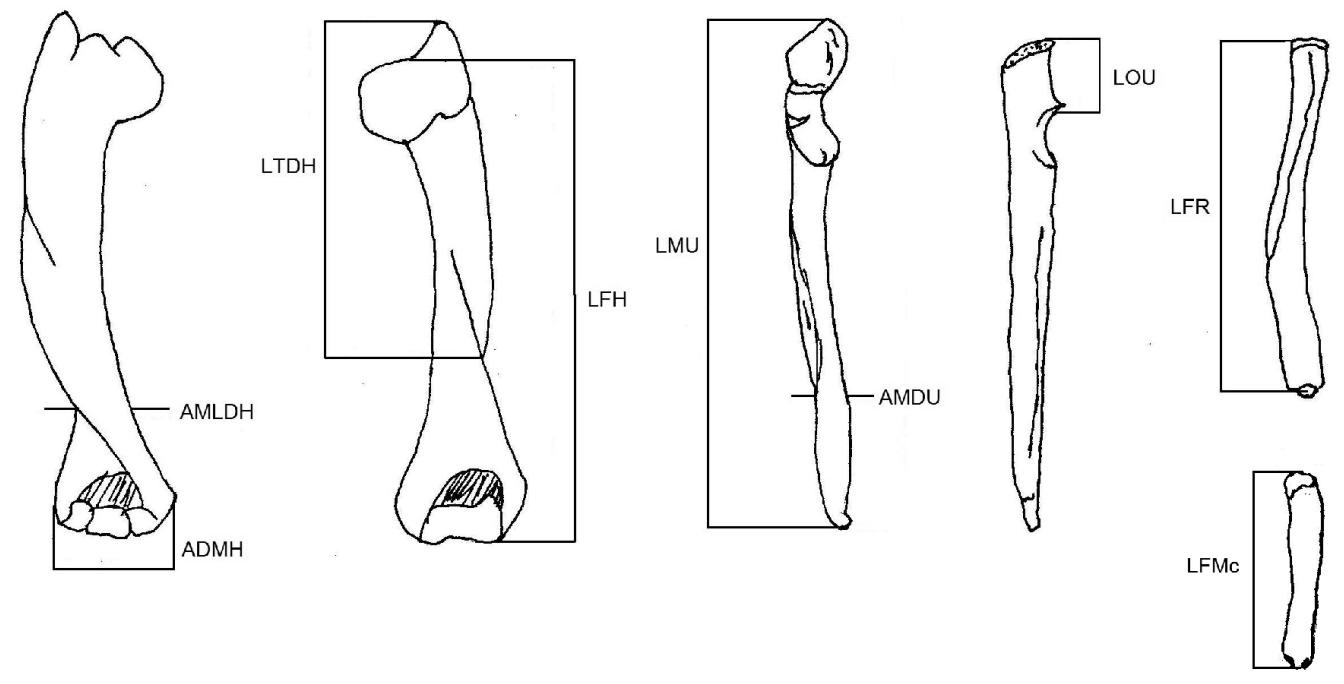

a.- Miembro anterior $\boldsymbol{D}$. branickii. Anchura distal máxima (ADMH), Anchura mínima lateral de la diáfisis (AMLDH), Longitud fisiológica del húmero (LFH), Largo tuberosidad deltoidea (LTDH), Longitud máxima de la ulna (LMU), Largo del olecranon (LOU), Anchura mínima lateral de la diáfisis (AMDU), Longitud fisiológica del radio (LFR), Longitud fisiológica del metacarpo (LFMc)
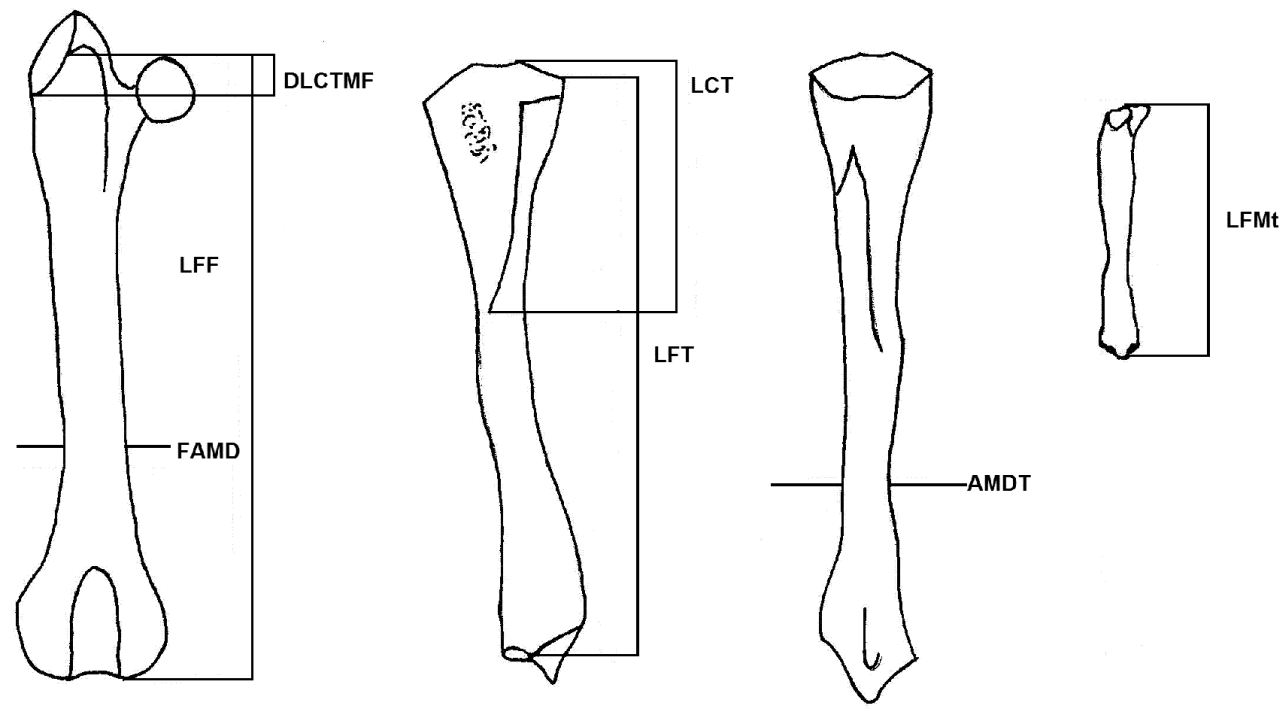

b.- Miembro posterior $D$. branickii. Anchura mínima de la diáfisis (FAMD), Longitud fisiológica del fémur (LFF), Distancia de la cabeza del fémur hasta el trocánter mayor, Longitud fisiológica de la tibia (LFT), Anchura mínima de la diáfisis (AMDT), Largo de la Cresta (LCT), Longitud fisiológica del metatarso (LFMt)

Figura 1. Esquema de las medidas obtenidas para el esqueleto apendicular.

pronator y supinator del antebrazo; es considerado, también, como un buen indicador del hábito de cavar (Hildebrand et al. 1985; Lessa E Stein, 1992).

- Índice de la robustez de húmero (IRH): AMLDH/LFH $\mathrm{x} 100$.
- Índice de la robustez de la ulna (IRU): AMDU/LFU x 100. Es un indicador de la robustez del miembro anterior y el ancho relativo, disponible para la inserción de los músculos involucrados con la pronación y supinación del miembro y la flexión de la mano y los dígitos. 
- Índice gluteal (IG): LCTMF/LFF x 100. Muestra la ventaja mecánica del músculo glúteo medio y la velocidad de la extensión femoral.

- Índice crural (IC): LFT/LFF x 100. Determina la capacidad del miembro posterior para alcanzar velocidades mayores basándose, en la extensión del mismo.

- Índice de la robustez femoral (IRF): AMLDF/LFF x 100. Define la capacidad para soportar la masa corporal y para resistir fuerzas verticales asociadas con el aumento de velocidad (Biewener $\mathcal{E}$ Taylor, 1986; Demes et al. 1994).

- Índice de la robustez de la tibia (IRT): AMLDT/LFT x 100. Es un indicador de la fuerza de la pierna y del ancho relativo para el origen de los músculos que actúan sobre el tobillo.

- Índice de la cresta de la tibia (ICT): LCT/LFT x 100. Determina la fuerza de la pierna y de la anchura relativa disponible para la inserción de los músculos gracilis, semitendinoso, semimembranoso y de los flexores del pie (Elftman, 1929).

- Índice intermembral (II): Se calcula a partir de las longitudes fisiológicas de los miembros anterior y posterior (LFH+LFR+LTMC)/(LFF+LFT+LTMT); refleja la predominancia del miembro anterior sobre el posterior.

Los datos, se analizaron mediante estadística descriptiva y después de evaluada la distribución normal, se aplicó una prueba de t de Student, para establecer diferencias, estadísticamente significativas, entre los valores obtenidos por especie y entre especies.

\section{RESULTADOS Y DISCUSIÓN}

Los promedios de los valores morfométricos obtenidos para los huesos del esqueleto apendicular de las dos especies indican las diferencias esperadas (Figura 2), por ellas poseer masas corporales distintas. D. branickii es una especie más robusta (Osbahr, 1998b), y el peso promedio obtenido para los ejemplares en estudio es mayor que el de C. taczanowskii (Tabla 1). Es así, como se registran diferencias interespecíficas entre las longitudes fisiológicas de los huesos largos que conforman el miembro anterior $\left(\mathrm{p}<0,001 ; \mathrm{t}_{(\mathrm{LHF})}=13,50 ;\right.$ g.l. $=7$; $\mathrm{t}_{(\llcorner M U)}=14,16 ; g . l .=6 ; \mathrm{t}_{(L F R)}=12,43 ;$ g.l. $\left.=6\right)$, mientras que el metacarpo no presenta dichas diferencias $(p>0,1$; $\left.\mathrm{t}_{(\mathrm{LFMC})}=0,17 ; \mathrm{g} . \mathrm{l} .=3\right)$. Esta diferencia, estadísticamente significativa, se observa para las longitudes fisiológicas, incluyendo el metatarso, de los huesos que componen el miembro posterior $\left(\mathrm{p}<0,001 ; \mathrm{t}_{(\mathrm{LFF})}=6,59 ;\right.$ g.l. $=7$; $\left.\mathrm{t}_{(\mathrm{LFT})}=9,48 ; g . l .=7 ;(\mathrm{p}<0,01) ; \mathrm{t}_{(\mathrm{LFMT})}=6,55 ; \mathrm{g} . \mathrm{l} .=4\right)$. De igual manera, se observa que en ambas especies, en promedio, la longitud total del miembro anterior (LTMA) es menor que la del posterior (LTMP) (Figura 3), existiendo diferencias, estadísticamente significativas, tanto interespecíficas LTMA $\mathrm{t}_{(\mathrm{C} \text {. taczanowskii, } \text {. branickii) }}=10,47$;

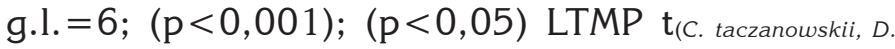
branickii) $=2,46 \mathrm{~g} . \mathrm{l} .=7)$ como intraespecíficas $(\mathrm{p}<0,001)$; C. taczanowskii $\mathrm{t}_{(\mathrm{LTMA}, \mathrm{LTMP})}=8,75 ; \mathrm{g} . \mathrm{l} .=6 ;(\mathrm{p}<0,02) ; D$. branickii $_{(\text {LTMA, LTMP) }}=2,85$; g.l. $\left.=18\right)$.

Entre los mamíferos, las especies más grandes tienden a ser más rápidas que las pequeñas; sin embargo, en el caso de los roedores, la velocidad es independiente de la masa corporal (Garland, 1983). Se esperaría que individuos más pesados se muevan más lento que individuos livianos, debido a que su masa elevada impide la función locomotora. En cambio, individuos que son estructuralmente grandes, posiblemente, se movilizan más rápido que individuos pequeños, porque presentan miembros largos y, por ende, registran una mayor amplitud de paso (Schulte-Hostedde \& Millar, 2002).

Existe una estrecha relación entre la morfología animal y su comportamiento, originando que las especializaciones morfológicas puedan repercutir en una mayor eficiencia durante determinada actividad (Hildebrand et al. 1985; Lammers E German, 2002). Los resultados aquí obtenidos permiten inferir diferencias entre las dos especies durante la locomoción y, por lo tanto, durante los patrones de uso del hábitat. Se puede deducir que al ser $D$. branickii un animal plantígrado presenta metatarsos más largos, característica que es notoria cuando se desplaza en marcha lenta, o durante los períodos de alimentación, cuando toma una postura semi-sentada ejerciendo un mayor apoyo sobre los miembros posteriores (Goeldi, 1904; Mohr, 1937; Collins E Eisenberg, 1972; Woods, 1984; Osbahr, 2000).

C. taczanowskii cuenta con metatarsos más cortos lo cual, podría coincidir con las características descritas por Mondolfi (1972), es decir, es una especie digitígrada, lo cual, le permite desplazarse a velocidades mayores, por existir un menor tiempo de contacto con el suelo, lo que 


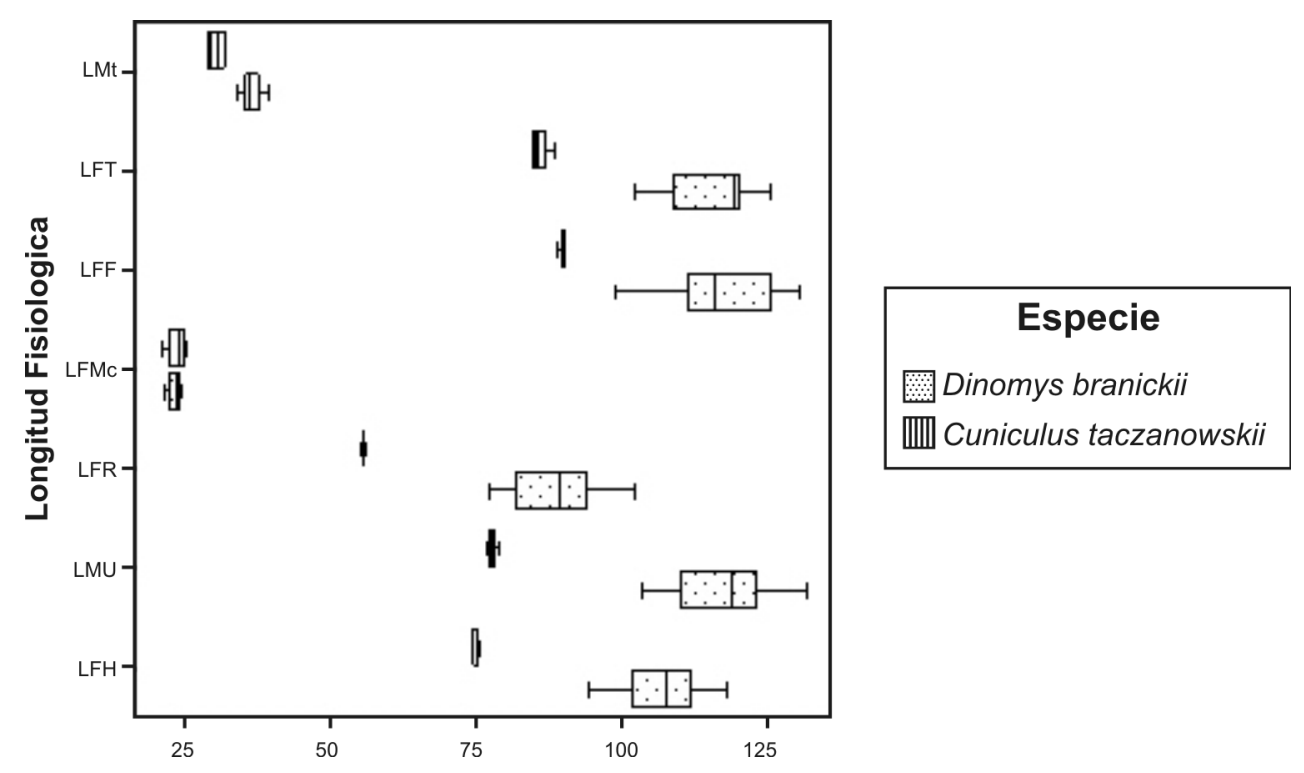

Figura 2. Valores promedio de las medidas morfométricas del esqueleto apendicular por especie. Las siglas empleadas equivalen a las mencionadas en la tabla 2.

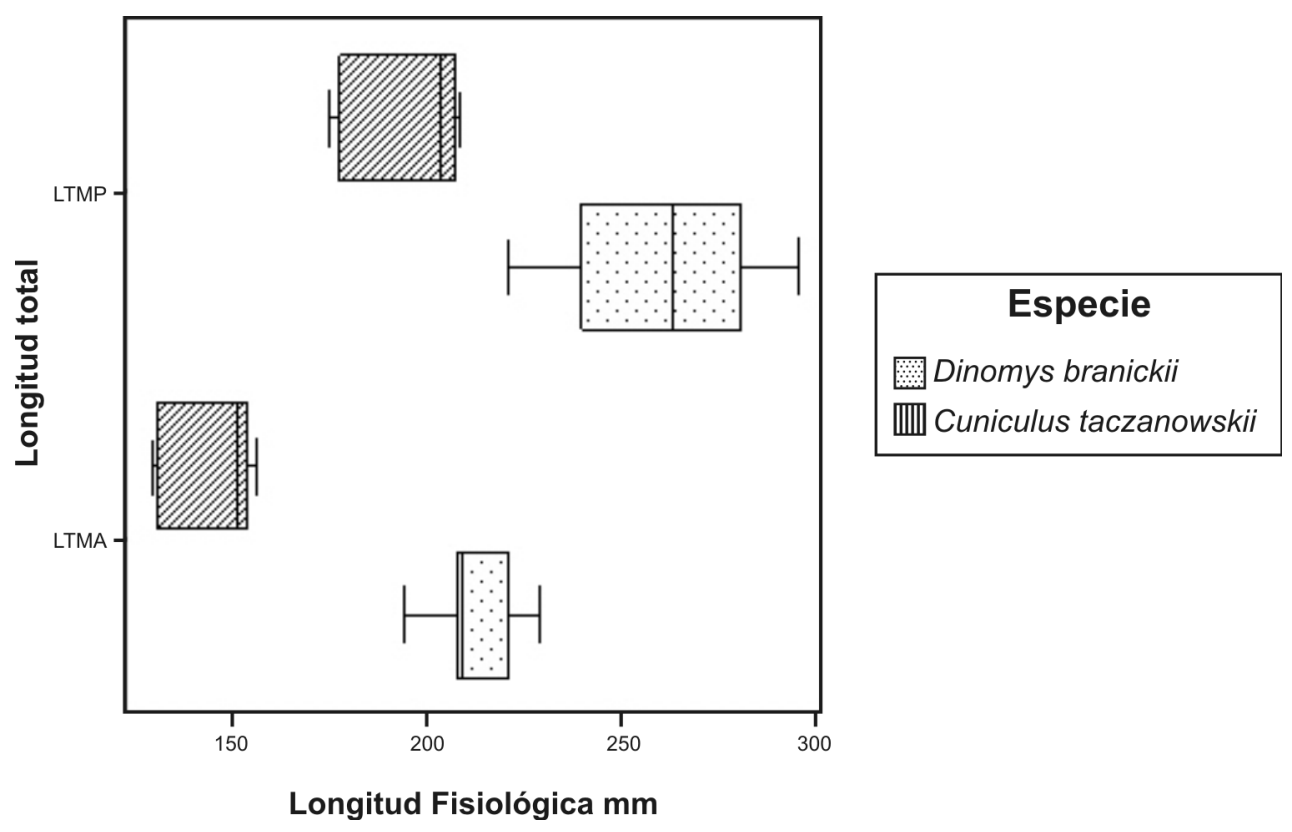

Figura 3. Valores promedio de la longitud del miembro anterior (LTMA) y posterior (LTMP) por especie.

se traduce en una fase de balanceo (swing fase) más prolongada (Espinosa et al. 2008). Esta situación faculta al animal para almacenar mayor energía elástica para que en el momento de apoyo, la fuerza y la velocidad de propulsión, se incrementen (Cavagna et al. 1964; Goslow et al. 1973; Alexander, 1988; 2002).
Existe un mayor desempeño en el trabajo de excavación para la adecuación de madrigueras por parte de $C$. taczanowskii; requiere una mayor fuerza y longitud en los dedos, puesto que debe remover cantidades considerables de tierra. D. branickii manifiesta momentos más esporádicos de excavación, ya que, 
preferencialmente, emplea como refugio cuevas naturales entre las rocas sin ampliarlas o adecuarlas (López et al. 2000; Osbahr, 1998a).

En C. taczanowskii, la diferencia entre la longitud del miembro anterior y el posterior genera una posición ligeramente inclinada de la columna vertebral, cuando el animal se encuentra en posición cuadrúpeda. Esta condición, le permite escabullirse en espacios muy reducidos, como cuevas de otros animales más pequeños, que adapta y habita durante largos periodos (Nowak \& Paradiso, 1999). En cambio, al caminar, la posición del lomo en $D$. branickii es paralela al suelo y su ingreso a las cuevas tiende a ser en posición caudal, como posible mecanismo de defensa ante los depredadores, debido a que le permite mostrar sus fuertes incisivos, mientras se encuentra en una posición desfavorable (Osbahr, 2000). Siendo adicionalmente un animal trepador, al descender de lugares elevados utiliza de igual manera la posición caudal, para lo cual, con las extremidades posteriores más largas, puede ejercer la propulsión necesaria para sujetarse a troncos u otras superficies inclinadas. De otra parte, esta característica morfológica -miembros anteriores menores que los posteriores-, se encuentra también en otros roedores caviomorfos, al igual que en pequeños ungulados (Tragulidae y Cephalophinae), así como en algunos marsupiales del género Macropus (wallabies). De acuerdo con Dubost (1968), Bourlière (1973) y Kingdon (1989) es una adaptación, común a diversos grupos de mamíferos pequeños, que habitan entre los arbustos de los bosques tropicales del mundo.

Debido a que, en general, para ambas especies los miembros anteriores son más cortos que los posteriores, se vislumbra un efecto sobre la longitud del paso y, por ende, sobre la velocidad de desplazamiento (Espinosa et al. 2008). A medida que la velocidad aumenta, el miembro anterior se ve obligado a incrementar sus movimientos, mientras que el miembro posterior aumenta la extensión de sus articulaciones (RochaBarbosa et al. 2005). Según Biknevicius (1993), los miembros posteriores mantienen una relación más estrecha con la capacidad de locomoción de los animales frente a los miembros anteriores, pues estos, además, se encuentran comúnmente relacionados con otro tipo de funciones, como la excavación o la manipulación de la comida, presentando adaptaciones que facilitan este tipo de comportamientos. Para el caso de D. branickii, los miembro posteriores más largos, derivados de patas más largas, permiten pasos más largos, además, que la especie presenta una cola más larga, que actúa como un órgano de balance y permite cambios en la dirección del cuerpo durante la fase de suspensión (Hildebrand E Goslow, 2001).

Al evaluar los valores promedio de los índices morfológicos calculados a partir de la medidas morfométricas obtenidas (Figura 4), se obtuvieron diferencias, estadísticamente significativas, entre las dos especies para el índice braquial $\left(p<0,001 ; t_{(B)}=14,05 ;\right.$ g.l. $\left.=6\right)$ y el índice de la robustez del fémur $\left(\mathrm{p}<0,01 ; \mathrm{t}_{\text {(IRF) }}=4,32 ; \mathrm{g} . \mathrm{l} .=7\right)$, con valores promedio mayores para $D$. branickii. Los índices del movimiento del hombro $\left(\mathrm{p}<0,05 ; \mathrm{t}_{(\mathrm{IMH})}=2,46\right.$; g.l. =7), de la habilidad fosorial $\left(\mathrm{p}<0,001 ; \mathrm{t}_{(\mathrm{IHF})}=12,74\right.$; g.l.=6), epicondilar $\left(p<0,01 ; t_{(\mathbb{E})}=4,46 ;\right.$ g.l. $\left.=7\right)$, de la robustez de la ulna $\left(\mathrm{p}<0,001 ; \mathrm{t}_{(\mathrm{IRU})}=9,94 ; \mathrm{g} . \mathrm{l} .=6\right)$ y de la cresta de la tibia $\left(\mathrm{p}<0,001 ; \mathrm{t}_{\text {(ICT) }}=56,02 ; \mathrm{g} . \mathrm{l} .=7\right)$, igualmente mostraron diferencias, estadísticamente significativas, pero con valores promedio mayores para C. taczanowskii. Los índices crural (IC), gluteal (IG), de la robustez de la tibia (IRT) e intermembranosos (II), no registraron diferencias, estadísticamente significativas, para las dos especies $\left(\mathrm{p}>0,1 ; \mathrm{t}_{(\mathrm{IC})}=1,62 ; \mathrm{t}_{(\mathrm{IG})}=0,67\right.$; $\mathrm{T}_{(\mathrm{IRT})}=0,11 ; \mathrm{t}_{(\mathrm{II})}=0,21 ;$ g.l. $\left.=7\right)$.

Según Vizcaíno et al. (1999) y Vizcaíno \& Milne (2002), así como también Elissamburu \& Vizcaíno (2004), el índice braquial (IB) equivale al largo funcional del miembro anterior y representa la capacidad de realizar movimientos más rápidos, puesto que considera el largo total del miembro anterior, de tal manera que un valor más elevado en el índice implica una capacidad de recorrer distancias a velocidades mayores (animales cursoriales). Sin embargo aquellos que presentan adaptaciones para cavar (fosoriales) reflejan valores más bajos, sugiriendo que poseen los miembros anteriores más cortos, es decir, que las diferencias observadas para el índice braquial en las dos especies (figura 4) reflejan nuevamente que $D$. branickii, aunque presenta una amplia gama de hábitos trepadores (Weisbecker E Schmid, 2007), tiene una mayor capacidad cursorial que $C$. taczanowskii.

Un indicador de la fuerza de palanca del húmero es el índice del movimiento del hombro (IMH), el cual, se encuentra más elevado en especies que poseen adaptaciones para cavar. Además existe una estrecha relación con el índice de habilidad fosorial (IHF), que 

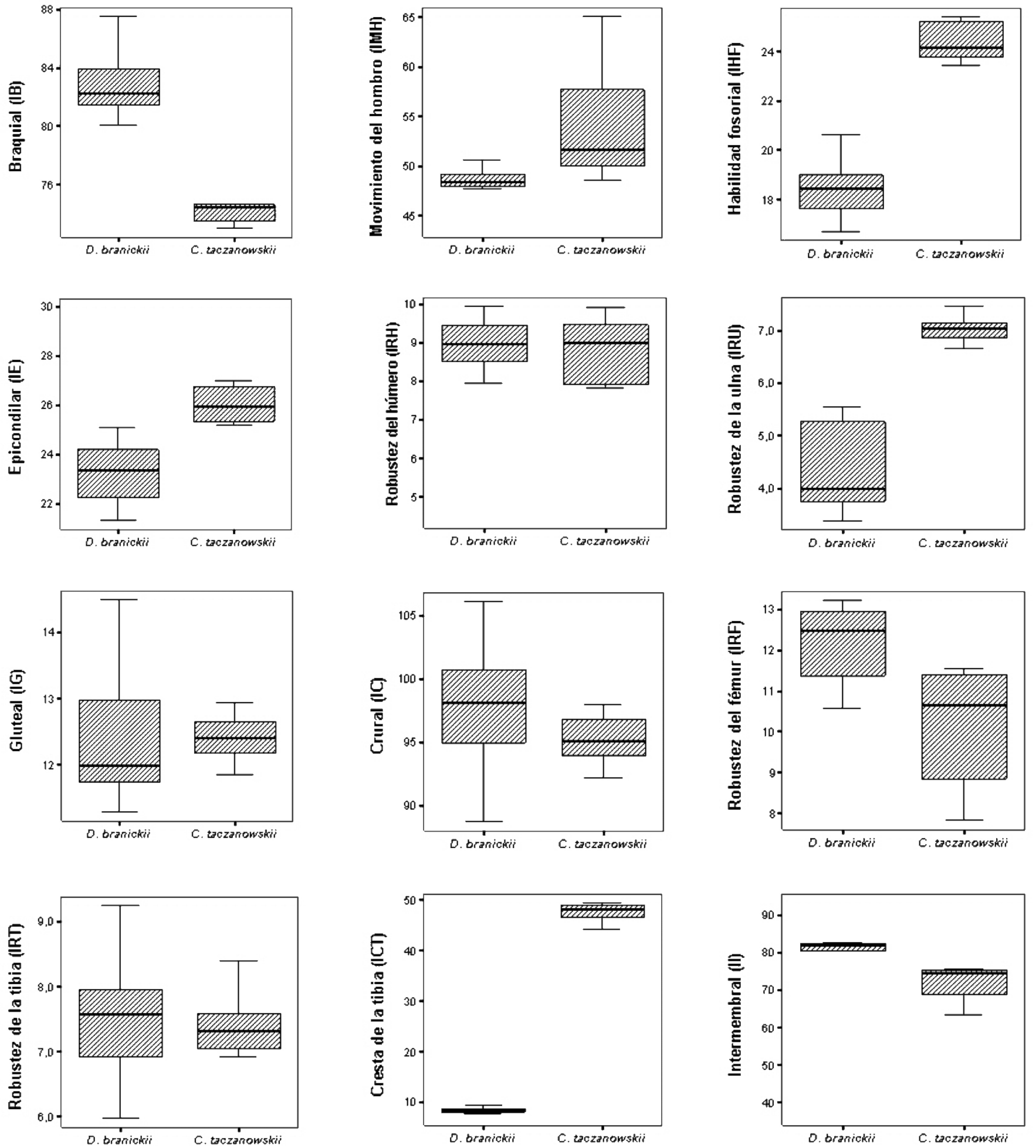

Figura 4. Diagramas de caja indicando cada índice promedio por especie. 
es estudiado de acuerdo a la longitud del olécranon y, permite determinar la fuerza de palanca que puede ejercer la ulna. De acuerdo con esto, son índices que establecen que los animales con valores elevados poseen una capacidad de fuerza mayor en los miembros anteriores, lo que les proporciona ventajas mecánicas durante la excavación, mientras que los animales con valores más bajos, generalmente, logran alcanzar velocidades más altas durante el desplazamiento (Vizcaíno et al. 1999; Vizcaíno E Milne, 2002; Elissamburu E Vizcaíno, 2004), de tal manera que los valores promedio mayores para IMH e IHF (figura 4) obtenidos para C. taczanowskii explican, nuevamente, la alta capacidad para realizar movimientos de palanca con el miembro anterior, durante la remoción de tierra, al ingresar a espacios reducidos; esta situación se ve reforzada por el hecho de poseer un mayor índice epicondilar (IE). Una mayor área entre los epicóndilos posibilita una mayor inserción muscular durante los movimientos de flexión, de pronación y de supinación, así como también en la fuerza que posiblemente pueda ser empleada por la mano, lo que facilita movimientos para la excavación. En animales con modificaciones más especializadas, dirigidas hacia la excavación, el índice de la robustez de la ulna (IRU) es un indicador de la capacidad que tiene el hueso para posibilitar una mayor inserción muscular, ya que esto les permite ejercer una mayor fuerza durante la flexión de las muñecas y de los dedos (Hildebrand et al. 1985). Esta condición explica los valores más elevados obtenidos para el caso de $C$. taczanowskii (Figura 4) reforzando una característica morfofisiológica que le otorga una ventaja durante su desempeño en la excavación.

Los índices obtenidos en el miembro posterior fueron similares entre ambas especies para el gluteal (IG), el crural (IC) y la robustez de la tibia (IRT) (Figura 4). IG representa la eficiencia de los músculos glúteos y, generalmente, se puede relacionar, al igual que IC, con la adaptación de los miembros para la velocidad, mientras que IRT es un indicador de la capacidad de soporte y de propulsión del cuerpo, durante la locomoción. De acuerdo con Howell (1944); Fleagle (1979) y Vizcaíno E Milne (2002), en animales cursoriales, se deben encontrar valores más elevados para IRT, condición que coincide con los valores promedio mayores obtenidos para este índice en $D$. branickii. El índice de la robustez de fémur (IRF) brinda una ventaja mecánica para la inserción muscular, así como también una rigidez mayor en el hueso, permitiéndole al animal una mayor fuerza en sus miembros posteriores; esto es importante en animales trepadores, ya que necesitan apoyarse sobre sus miembros posteriores, como es el caso de $D$. branickii. Según Biknevicius (1993), los valores más elevados se encuentran, posiblemente, relacionados con una mayor rigidez del fémur, lo cual, permitiría al animal alcanzar mayores velocidades durante la locomoción, debido a que una anchura mayor está relacionada a huesos más largos.

Durante la flexión del miembro posterior, en la primera fase del paso, el índice de la cresta de la tibia (ICT) es importante, ya que la inserción proximal se encuentra estrechamente relacionada con una mayor velocidad durante el movimiento propulsor inicial. Las especies que presentan los valores más bajos poseen comportamientos, en su mayoría, cursoriales (Elftman, 1929), como es el caso de D. branickii. En el caso de C. taczanowskii, se puede inferir que los valores tan elevados que presenta están relacionados con la capacidad de saltar (Mondolfi, 1972; Fernández, 1988), hasta 1,50m de altura, lo cual, puede reducir la diferencia que existe entre los valores estadísticos encontrados frente a los presentados por $D$. branickii. Los animales que poseen una mayor dominancia en sus miembros posteriores tienden a presentar valores más elevados del índice intermembral (II) (Viscaíno E Milne, 2002), que, de igual manera, aplica para animales de mayor tamaño, explicando que $D$. branickii registre valores superiores.

De acuerdo con los resultados obtenidos es posible sugerir que ambas especies tienen la misma capacidad de velocidad reflejada en los índices gluteal, crural y de la robustez de la tibia; sin embargo, se encuentran diferencias marcadas en la fuerza a nivel del fémur, siendo $D$. branickii, el que presenta los valores más elevados. Cabe recordar que esta especie muestra un uso importante de sus miembros posteriores, no solamente por ser animales plantígrados, sino por el hecho de ejercer mayor fuerza sobre los miembros posteriores al trepar y al alimentarse en posición sentada.

\section{CONCLUSIONES}

Aun cuando las especies en estudio registraron las diferencias esperadas entre las longitudes fisiológicas de los huesos largos que conforman el miembro anterior y posterior, sobresalen especialmente los metacarpos y 
metatarsos, explicando la locomoción digitígrada de $C$. taczanowskii y la condición plantígrada de $D$. branickii.

La diferencia entre la longitud de los miembros anteriores con respecto a los posteriores permite vislumbrar un efecto sobre la longitud del paso y, por ende, sobre la velocidad de desplazamiento.

Los índices morfológicos obtenidos corroboran las características anatómicas que corresponden a animales con capacidades para cavar, como es el caso de $C$. taczanowskii, al igual que la versatilidad que caracteriza a $D$. branickii durante la locomoción, al encontrarse equipado morfológicamente para desempeñarse durante la carrera y para trepar en búsqueda de alimento.

\section{AGRADECIMIENTOS}

Los autores agradecen a la ZGAP (Zoologische Gesellschaft für Arten und Populationsschutz), a la ZooAG Bielefeld, al Tierpark Cottbus, a los Zoológicos de Newquay, Chester y de Los Angeles por el permanente apoyo rebicido durante el desarrollo del presente trabajo.

\section{BIBLIOGRAFÍA}

ALEXANDER, R. 1988. Elastic mechanism in animal movement. Cambridge: Cambridge University Press. 185p.

ALEXANDER, R. 2002. Tendon elasticity and muscle function. Comp. Biochem. Physiol. A comp. Physiol. 133:1001-1011.

BACIGALUPE, L.D.; IRIARTE-DÍAZ, J.; BOZINOVIC, F. 2002. Functional morphology and geographic variation in the digging apparatus of cururos (Octodontidae: Spalacopus cyanus) J. Mammalogy. 83(1):145-152.

BIEWENER, A.A.; TAYLOR, C.R. 1986. Bone strain: a determinant of gait and speed? J. Exp. Biol. 123:382-400.

BOURLIÈRE, F. 1973. The comparative ecology of rain forest mammals in Africa and Tropical America: Some introductory remarks. In: Meggers, B.J.;
Ayensu, E.S.;Duckworth, W.D.; Smith. Inst. Press, Trop.Forest Ecosyst. in Africa and South America: A comparative review: p.279-292.

BIKNEVICIUS, A.R. 1993. Biomechanical scaling of limb bones and differential limb use in caviomorph rodents. J. Mammalogy. 74:95-107.

CAVAGNA, G.A.; SAIBENE, F.P.; MARGARIA, R. 1964. Mechanical work in running. J. Appl. Physiol. 19:249-256.

CHRISTIANSEN, P. 1999. Scaling of limb long bones to body mass in terrestrial mammals. J. Morphology. 239:167-190.

COLLINS, L.; EISENBERG, J. 1972. Note on the behavior and breeding of pacaranas (Dinomys branickii) in captivity. International Zoo Yearbook 12:108-114.

DEMES, B.; LARSON, S.G.; STERN, J.T. Jr.; JUNGERS, W.L.; BIKNEVICIUS, A.R.; SCHMITT, D. 1994. The kinetics of primate quadrupedalism: "Hindlimb drive" reconsidered. J. Human Evol. 26:353-374.

DUBOST, G., 1968. Les niches écologiques des forêts tropicales sud-américaines et africaines, souces de convergences remarquables entre rongeurs et artiodactyles. La terre et la vie 22:3-28.

ELFTMAN, H.O. 1929: Functional adaptations of the pelvis of marsupials. Bull. Am. Museum of Natural History. 63:189-232.

ELISSAMBURU, A.; VIZCAÍNO, S.F. 2004. Limb proportions and adaptations in caviomorph rodents (Rodentia: caviomorpha). J. Zool. 262:145-159.

ENDO, H.; SASAKI, M.; HAYASHI, Y.; KOIE, H.; YAMAYA, Y.; KIMURA, J. 2001. Carpal bone movements in gripping action of the giant panda (Ailuropoda melanoleuca). J. Anatomy. 198:243-246.

ESPINOSA, D.; VILLAMIZAR, A.; OSBAHR, K.; ACEVEDO, P. 2008. Caracterización del desplazamiento en cautiverio de dos especies de roedores histricomorfos (Cuniculus taczanowskii y Dinomys 
branickii) mediante el estudio morfofisiológico comparado de los miembros anterior y posterior de cada especie. Trabajo de grado, Fac. Medicina Veterinaria, U. Ciencias Aplicadas y Ambientales U.D.C.A Bogotá. 72p.

FERNÁNDEZ, B.A. 1988. Agouti taczanowskii En: Mamíferos en Venezuela. Lista y claves para la identificación. Primera Edición. ASOVEN. Maracay - Venezuela. p.129-130, 142, 183-185.

FERNÁNDEZ, M.E.; VASSALLO, A.Y.; ZARATE, M. 2000. Functional morphology and palaeobiology of the Pliocene rodent Actenomys (Caviomorpha: Octodontidae): The evolution to a subterranean mode of life. Biology J. Linnean Soc. 71:71-90.

FLEAGLE, J.G. 1979. Primate adaptation and evolution. New York: Academic Press. 486p.

FLETCHER, T.F. 2007. Anatomical adaptations for cursorial locomotion and impact of diet. Disponible desde Internet en: http://vanat.cvm.umn.edu/run (con acceso 20/05/08).

GARLAND, T. Jr. 1983. The relation between maximal running speed and body mass in terrestrial mammals. J. Zoology. London. 199:157-170.

GINSBURG, L. 1961. Plantigradie et digitigradie chez les carnivores fissipedes. Mammalia. 25:1-21.

GOELDI, E. 1904. On the rare rodent Dinomys branickii (Peters). Proc. Zool. Soc. London. 2:158-162.

GOSLOW, G.E.; REINKING, R.M. Jr.; STUART, D.G. 1973. The cat step cycle: hindlimb joint angles and muscle lengths during a restrained locomotion. J. Morphology. 141:1-42.

HILDEBRAND, M.; BRAMBLE, D.M.; LIEM, K.F.; WAKE, D.B. 1985. Digging in quadrupeds. In: Hildebrand, M.; Goslow, G. (eds.) Functional Vertebrate Morphology. Cambridge, MA, Belknap Press. p.89-109.

HILDEBRAND, M.; GOSLOW, G. 2001. Analysis of Vertebrate Structure. $5^{\text {th }}$ ed. New York. John Wiley E Sons. 660p.
HOWELL, B.A. 1944. Speed in animals. The specialization for running and leaping. Chicago: University of Chicago Press. 270p.

IRSCHICK, D.J. 2002. Evolutionary approaches for studying functional morphology: examples from studies of performance capacity. Integ. Comp. Biol. 42:278-290.

KIMURA, T. 2003. Diferentiation between fore - and hindlimb bones and locomotor behaviour in Primates. Folia Primatologica. 74(1):17-32.

KINGDON, J. 1989. East African mammals: An atlas of evolution in Africa. The University of Chicago Press, Vol III-C (Bovids), 393p.

LAMMERS, A.R.; GERMAN, R.Z. 2002. Ontogenetic allometry in the locomotor skeleton of specialized half-bounding mammals. J. Zool. London. 258:48-495.

LESSA, E.P.; STEIN, B.R. 1992. Morphological constrains in the digging apparatus of pocket gophers (Mammalia: Geomydae). Biology J. Linnean Soc. 47:439-453.

LOGUERCIO, M.F. 2005. Desempenho e comportamento locomotor da capivara, Hydrochaeris hydrochaeris (Linnaeus, 1766) (Rodentia: Cavioidea). Dissertação de Mestrado, Dept. Zool., U. do Estado do Rio de Janeiro, Brasil, 108p.

LÓPEZ, L.; LÓPEZ, I.; MORA J.; OSBAHR, K. 2000. Estudio preliminar del comportamiento de Dinomys branickii (Peters, 1873) en cautiverio. Rev. U.D.C.A Act. E Div. Cient. 3(1):28-35.

MOHR, E. 1937. Vom Pacarana. Dinomys branickii (Peters). Der Zoologische Garten (Neue Folge). 9:204-209.

ONDOLFI, E. 1972. Mamíferos de Caza en Venezuela. La Lapa o Paca. Defensa de la Naturaleza. 2:4-16.

NOWAK, R.M; PARADISO, J.L. 1999. Walker's mammals of the world. $6^{\text {th }}$ Edition. The Johns Hopkins University Press. 1936p. 
OSBAHR, K. 1998a. Guía para la conservación de la "guagua loba" o "pacarana" (Dinomys branickii). En: Bernal, H.Y.; Farfán, M. (eds). $1^{a}$ ed. SECAB, Ciencia y Tecnología. Convenio Andrés Bello. Bogotá. 65:1-43.

OSBAHR, K. 1998b. Determinación de algunos parámetros sanguíneos útiles como herramienta para la evaluación del hábitat natural de Agouti taczanowskii y Dinomys branickii. Rev. U.D.C.A Act. E Div. Cient. 1(2):68-76.

OSBAHR, K. 2000. Determinación de patrones de comportamiento de un grupo de guaguas lobas (Dinomys branickii) mantenido en semicautiverio. Libro de Resúmenes. Primer Congreso Nacional de Zoología. Universidad Nacional. Santafé de Bogotá. Colombia. p.31.

PINTO, M.; ZUÑIGA, H.; TORRES, O. 2002. Estudio Sistemático del Género Cavia Pallas, 1766 (Rodentia: Caviidae) en Colombia. Revisión del Registro Arqueológico Colombiano. Ed. Guadalupe Ltda. Bogotá Colombia. p.29-48.

QUESADA, R.; GLOOBE, H. 1988. Osteología del Tepezcuinte (Cuniculus paca): cinturas y miembros. Anatomy, Histology, Embriology. 17: 60 - 71.

ROCHA-BARBOSA, O.; RENOUS, S.; GASC, J.P. 1996. Adaptation à la course chez le cobaye Cavia porcellus (Mammifère, Rongeur, Caviomorphe). Bull. Soc. Zoologie, France, 121(1):121-123.

ROCHA-BARBOSA, O.; RENOUS, S.; GASC, J.P. 1996. Comparison of the fore and hind limbs kinematics in the symmetrical and asymmetrical gaits of a caviomorph rodent, the domestic guinea pig, Cavia porcellus (Linné, 1758) (Rodentia, Caviidae). Ann. Scienc. Nat., Zoologie, Paris, 17(4):149-165.

ROCHA-BARBOSA, O.; YOULATOS, D.; GASC, J.P.; RENOUS, S. 2002. The clavicular region of some cursorial Cavioidea (Rodentia:Mammalia). Mammalia, Paris, Françe. 66(3):413-421.

ROCHA-BARBOSA, O.; DE CASTRO, M.F.; RENOUS, S.; GASC, J.P. 2005. Limb joint kinematics and their relation to increasing speed in the guinea pig Cavia porcellus (Mammalia: Rodentia). J. Zool. London 266:293-305.

ROCHA-BARBOSA, O.; LOGUERCIO, M.F.; RENOUS, S.; GASC, J.P. 2007. Comparative study on the forefoot and hindfoot intrinsic muscles of some Cavioidea rodents (Mammalia, Rodentia). Zoology Jena, Alemania, 110:58-65.

SANTORI, R.T.; ROCHA-BARBOSA, O.; VIEIRA, M.V.; MAGNAM NETO, J. A.B.; LOGUERCIO, M.F. 2005. Locomotion in aquatic, terrestrial, and arboreal habitat of thick-tailed opossum, Lutreolina crassicaudata (DESMAREST, 1804). J. Mammal. North Carolina, USA. 86:902-908.

SANTORI, R.T.; VIEIRA, M.V.; ROCHA-BARBOSA, O.; MAGNAM NETO, J.A.B.; GOBBI, N. 2008. Water absorption of the fur and swimming behavior of semiaquatic and terrestrial Oryzomine rodents. J. Mammal. 89:1152-1161.

SCHULTE-HOSTEDDE A.I.; MILLAR, J.S. 2002. Measuring sexual size dimorphism in the yellow-pine chipmunk Tamias amoeus. Can. J. Zoology. 78:728-733.

TARABORELLI, P.; SASSI, P.; GIANNONI, S.M. 2007. Registro morfo-ecológico de Microcavia australis (Caviidae, Rodentia) en la puna de la provincia de San Juan, Argentina. Mastozool. Neotrop. 14(1):107-112.

VIZCAÍNO, S.F.; FARIÑA, R.A.; MAZZETTA, G. 1999. Ulnar dimensions and fossoriality in armadillos and other South American mammals. Acta Theriologica. 44.309-320.

VIZCAÍNO, S.F.; MILNE, N. 2002. Structure and function in armadillo limbs (Mammalia: Xenarthra: Dasypodidae). J. Zoology. 257:117-127.

WEISBECKER, V.; SCHMID, S. 2007. Autopodial skeletal diversity in hystricognath rodents: Functional and phylogenetic aspects. Mammalian Biology. 72(1):27-44. 
WOODS, C.A. 1984. Hystricognath Rodents. In: Ander-

Recibido: Enero 9 de 2009 son, S.; Jones J.K. Jr. (Eds.) Orders and Families Aceptado: Abril 29 de 2009 of Recent Mammals of the World. John Wiley and Sons. New York. p.389-446. 